Semiannual Progress Report, December--June 1974

NASA Grant NGR 16-002-038

NUMERICAL COMPUTATION OF

TWO-DIMENSIONAL VISCOUS BLUNT BODY

FLOWS WITH AN IMPINGING SHOCK

(NASA-CR-138308) NUMERICAL COMPUTATION OF

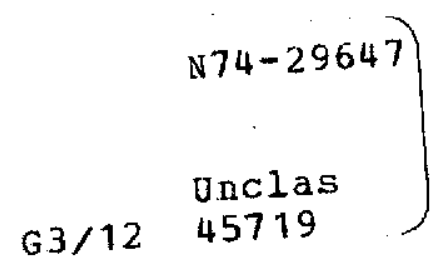
WITH AN IMRINGING SHOCK Semiannual progress (Iowa state Univ $\$ 4.00$ CSCL 200 and Technology)

Dr. John C. Tannehili

Department of Aerospace Engineering

Iowa State University of Science and Technology

Ames, Iowa 50010 


\title{
NUMERICAL COMPUTATION OF TWO-DIMENSIONAL VISCOUS \\ BLUNT BODY FLOWS WITH AN IMPINGING SHOCK
}

\author{
J. C. Tannehil1* and T. L. Holst ${ }^{\dagger}$ \\ Iowa State University, Ames, Iowa
}

An extraneous shock impinging on a blunt body in a hypersonic flow has been observed to greatly increase both the heat transfer rate and pressure near the impingement point. In fact, Hains and Keyes ${ }^{1}$ have measured peak heating rates up to 17 times the ordinary stagnation point rate and pressure peaks up to 8 times the freestream pitot pressure as a result of shock impingement. Flow fields of this type will occur on the Space Shuttle and other maneuverable re-entry vehicles.

The intense heating and high pressures occur over a small region where a disturbance, originating at the intersection of the impinging shock and bow shock, strikes the body. The disturbance may be a free shear layer, a supersontc jet, or a shock depending on the strength and location of the fmpinging shock and the shape of the body. Edney ${ }^{2}$ has described six different types of shock Interference patterns which can occur. The shock interference pattern which produces the maximum heating rates and pressures is Type IV, which is shown in Fig. 1. In this type of interference pattern, , the disturbance is a supersonic jet which is embedded in. the subsonic portion of the flow field.

Because of the very complicated nature of shock impingement flow fields, previous attempts ${ }^{2-5}$ at predicting the maximum heating rates and pressures have been Iimited to semiempirical approaches. In the present study, this

Assoctate Professor, Department of Aerospace Engineering

+ Research Associate, Department of Aerospace Engineering 
empiricism has been eliminated since the entire shock impingement flow field has been numerically computed. This was accomplished by using a "time-dependent", finite-difference method ${ }^{6}$ which solves the complete set of NavierStokes equations for a compressible flow. The major reason for using the "time-dependent" method is that the resulting unsteady Navier-Stokes equations are a mixed set of hyperbolic-parabolic equations for both subsonic and supersonic flows. As a result, a very complicated flow field, such as the one shown In Fig. 1 where both subsonic and supersonic regions are present, can be calculated as an initial-value problem. An additional advantage is that since Navier-Stokes equations are solved in a conservative manner, shocks are automatically allowed to form without previous knowledge of their location or even existence.

In the present two-dimensional analysis, the computational domain extends from the bow shock to the body and includes both the upper and lower sonic 11nes. For ease of computation, two coordinate transformations have been employed. The first transformation maps the physical plane into a rectangular computational plane bounded on the left by the bow shock and bounded on the right by the body. The second transformation stretches the computational grid in the direction normal to the body in order to permit a better resolution of the boundary layer.

MacCormack's finite-difference algorithm ${ }^{7}$ is used to solve the NavierStokes equations at each interior grid point. In order to determine the flow variables at the grid points along the leftmost boundary, the bow shock is treated as a moving discontinuity across which the Rankine-Hugoniot equations are applied. The location of the bow shock at each time step is determined using a predictor-corrector method ${ }^{6}$. All other shocks which exist between the bow shock and the body as well as any shear layers, jets, and the boundary layer are automatically "captured" in the finite-difference solution. 
Initially, the blunt body flow without the impinging shock is computed. The 1mpinging shock is then introduced into the flow fleld by specifying its location and strength. Thus, the freestream conditions behind the impinging shock are known. The computation is then restarted and is continued until the final "steady-state" solution is reached.

The present method has been used to compute the two-dimensional flow fields resulting when different shocks are allowed to impinge on a circular cylinder. The freestream conditions chosen for these calculations were

$$
\begin{array}{ll}
\mathrm{M}_{\infty}=4.6 & \mathrm{P}_{\infty}=14.93 \text { newtons } / \mathrm{m}^{2} \\
\operatorname{Re}_{D_{\infty}}=10,000 & \mathrm{~T}_{\infty}=167^{\circ} \mathrm{K} \\
\operatorname{Pr}=.72 & \mathrm{D}=.3048 \mathrm{~m} \\
\gamma=1.4 & \mathrm{~T}_{\text {wa11 }}=556^{\circ} \mathrm{K}
\end{array}
$$

A mesh consisting of 31 grid points in the normal direction and 51 grid points in the transverse direction was used in all computations. The Mach number contours for the blunt body flow without the impinging shock are shown in Fig. 2. The boundary layer is clearly evident in this figure. Computations were then made of two cases in which shocks of different strengths were allowed to impinge on the undisturbed flow field at a point near the stagnation streamline. These impinging shocks made angles of $16.1^{\circ}$ and $20.9^{\circ}$, respectively, with the freestream direction.

The Mach number contours for the $16.1^{\circ}$ shock impingement calculation are shown in Fig. 3. In this case, a shear layer emanates from the impingement point and makes a tangential approach to the body surface. This corresponds to a Type III interference pattern although no shock from the body surface is observed. This is probably due to the tangential approach of the shear layer. The Mach number contours for the $20.9^{\circ}$ shock impingement calculation are shown in Fig. 4. In this case a shear layer emanates from the impingement point and is intercepted by a shock wave which starts at the upper kink in 
the bow shock. This is a Type IV interference pattern. The internal shock wave is smeared as would be expected in a "shock-capturing" calculation. The impinging shock has caused the stagnation point to be moved a considerable distance from its original location (see Fig. 4). A comparison of the pressures and heat transfer rates on the body surface before and after impingement are shown in Figs. 5 and 6 . The impinging shock causes a peak pressure which is 2.4 times greater than the undisturbed stagnation point pressure and a peak heating rate which is 2.5 times greater than the undisturbed stagnation point rate.

The flow conditions and shock angles chosen for these sample calculations correspond with the tests of Edney ${ }^{2}$, which were three-dimensional flows resulting from a planar shock impinging on a body of revolution. Although the present calculations are two-dimensional, qualitative comparisons can still be made with the Edney experiments, espectally near the bow shock where the flow is locally two-dimensional. The correctness of the present calculations is inferred by these comparisons.

\section{References}

1. Hains, F. D. and Keyes, J. W., "Shock Interference Heating in Hypersonic Flows", AIAA Journa1, Vo1. 10, No. 11, Nov. 1972, pp. 1441-1447.

2. Edney, B., "Anomalous Heat Transfer and Pressure Distribution on Blunt Bodies at Hypersonic Speeds in the Presence of an Impinging Shock," FFA Rept. 115, 1968, The Aeronaut1cal Research Institute of Sweden, Stockholm, Sweden.

3. Keyes, J. W. and Hains, F. D., "Analytical and Experimental Studies of Shock Interference Heating in Hypersonic Flows," TN D-7139, 1973, NASA.

4. Morris, D. J. and Keyes, J. W., "Computer Programs for Predicting Superson1c and Hypersonic Interference Flow Fields and Heating," TM X-2725, 1973, NASA. 
5. Bertin, J. J. and Graumann, B. W., "Analysis of High Velocity, Real-Gas Effects on the Shock-Interference Pattern for Delta-Wing Orbiters," AIAA Paper No. 74-522, 1974.

6. Tannehil1, J. C. and Holst, T. L., "Numerical Computation of Two-Dimensional Viscous Blunt Body Flows with an Impinging Shock," ERI Rept. 74057, 1974, Iowa State Univers1ty, Ames, Iowa.

7. MacCormack, R. W., "Numerical Solution of the Interaction of a Shock Wave with a Laminar Boundary Layer," Lecture Notes in Physics, Vo1. 8, Springer-Verlag, New York, 1971, pp. 151-163. 
$6 x^{\prime \prime}$

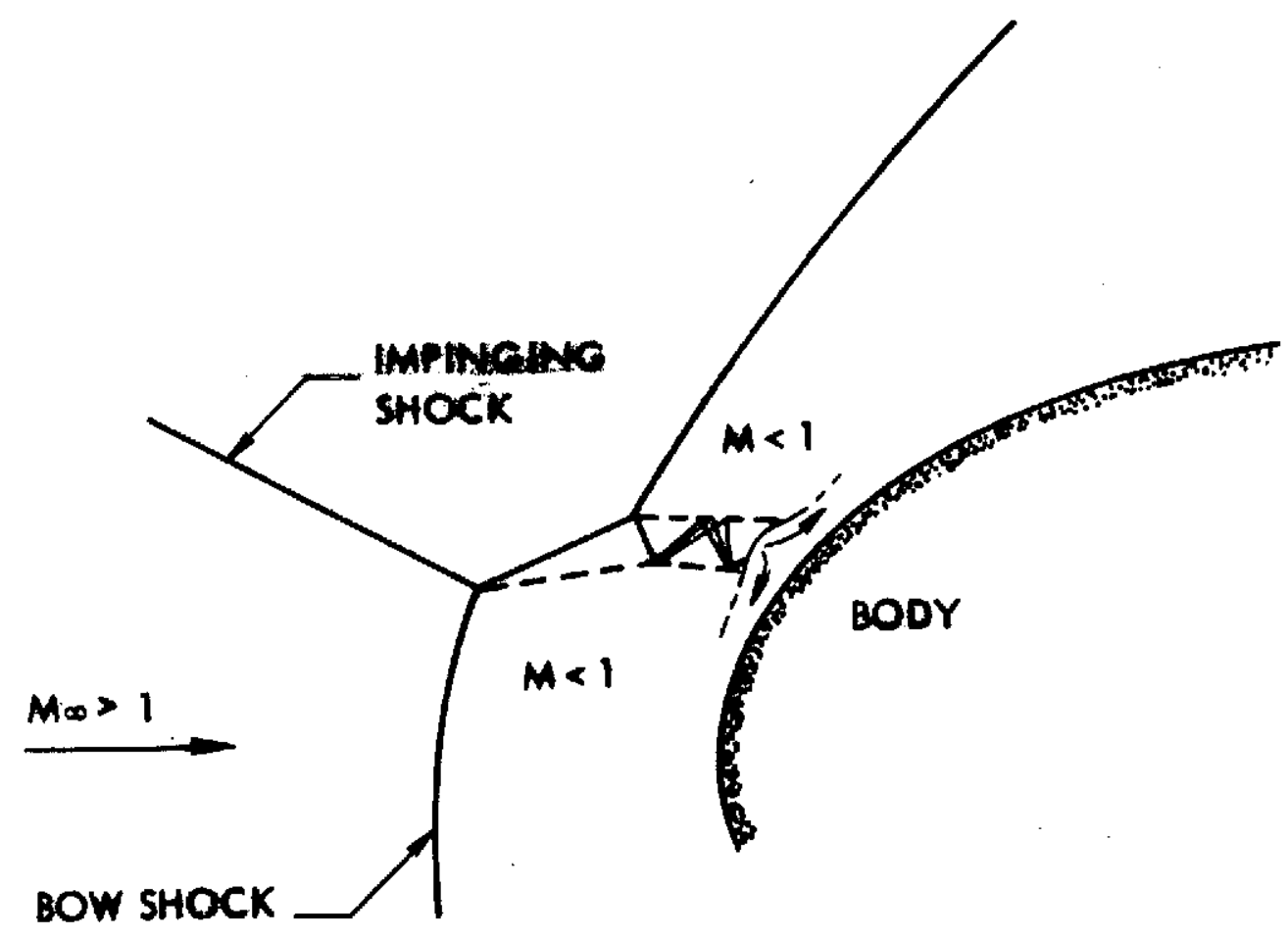

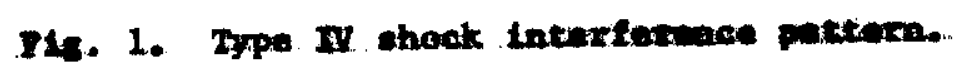




$$
C
$$




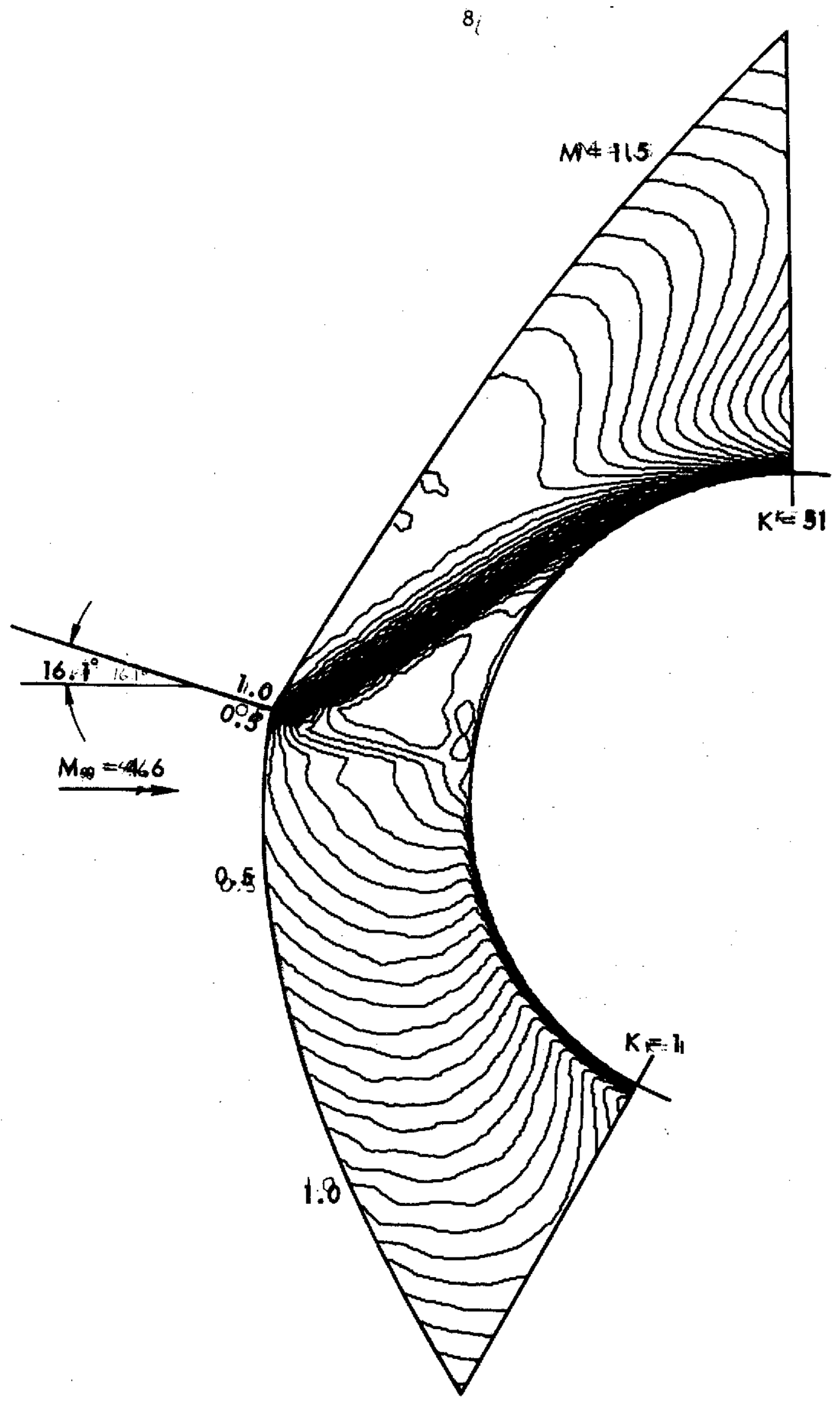

F19. 3. Mach number contours for $16.1^{\circ}$ shock impingement. 


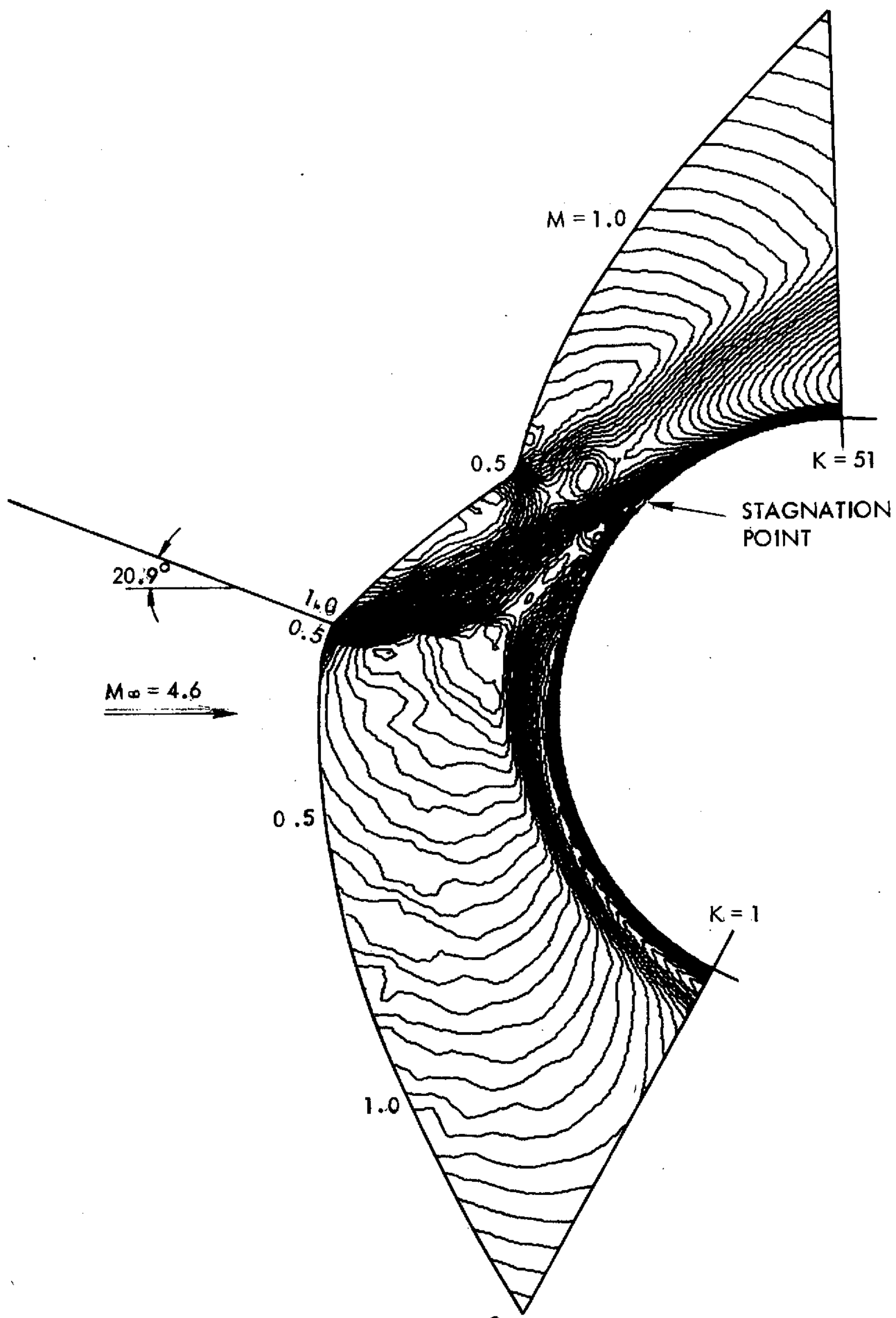

Fig. 4. Mach number contours for $20.9^{\circ}$ shock impingement. 


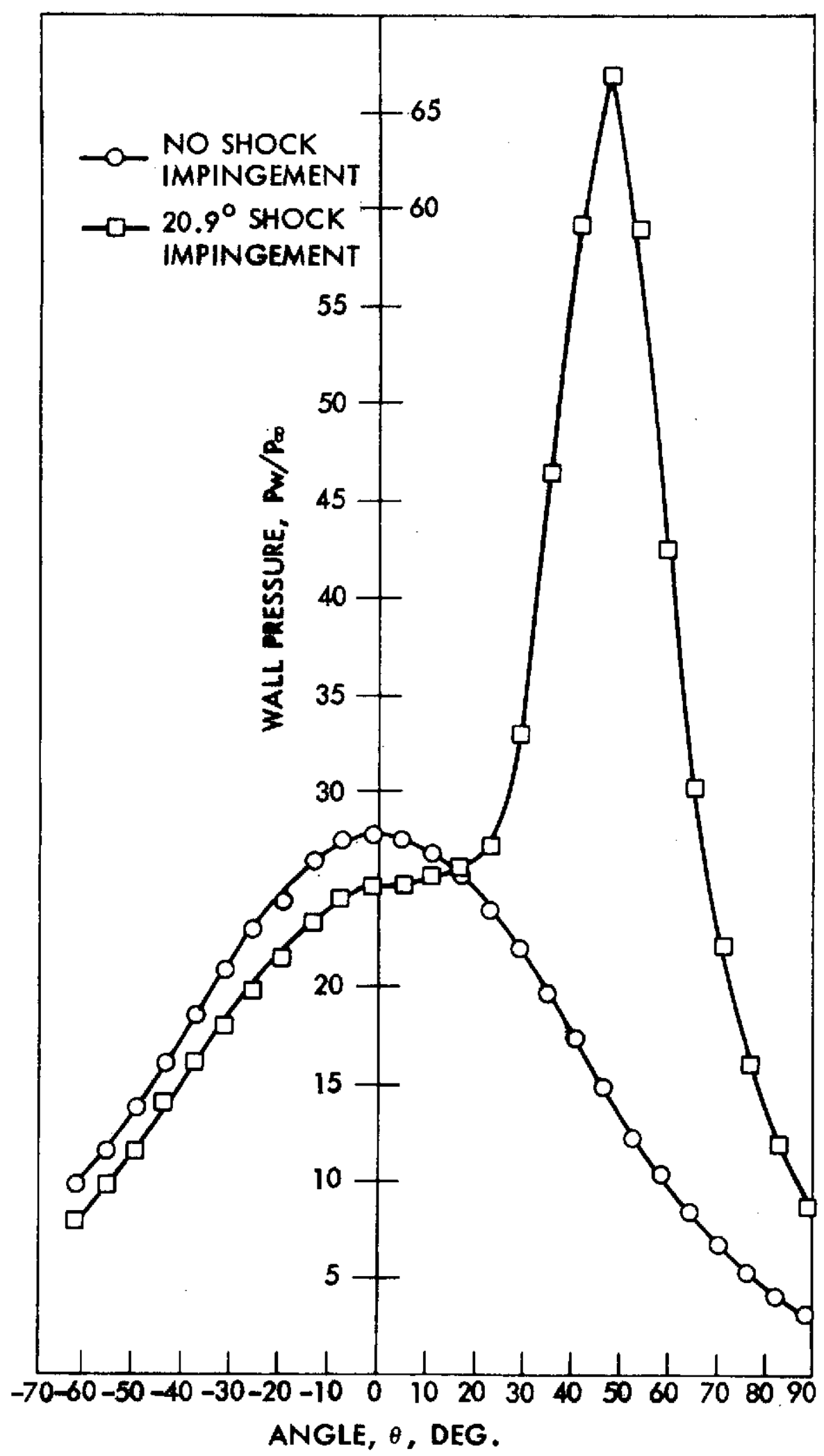

F18. 5. Comparison of wa11, pressures. 


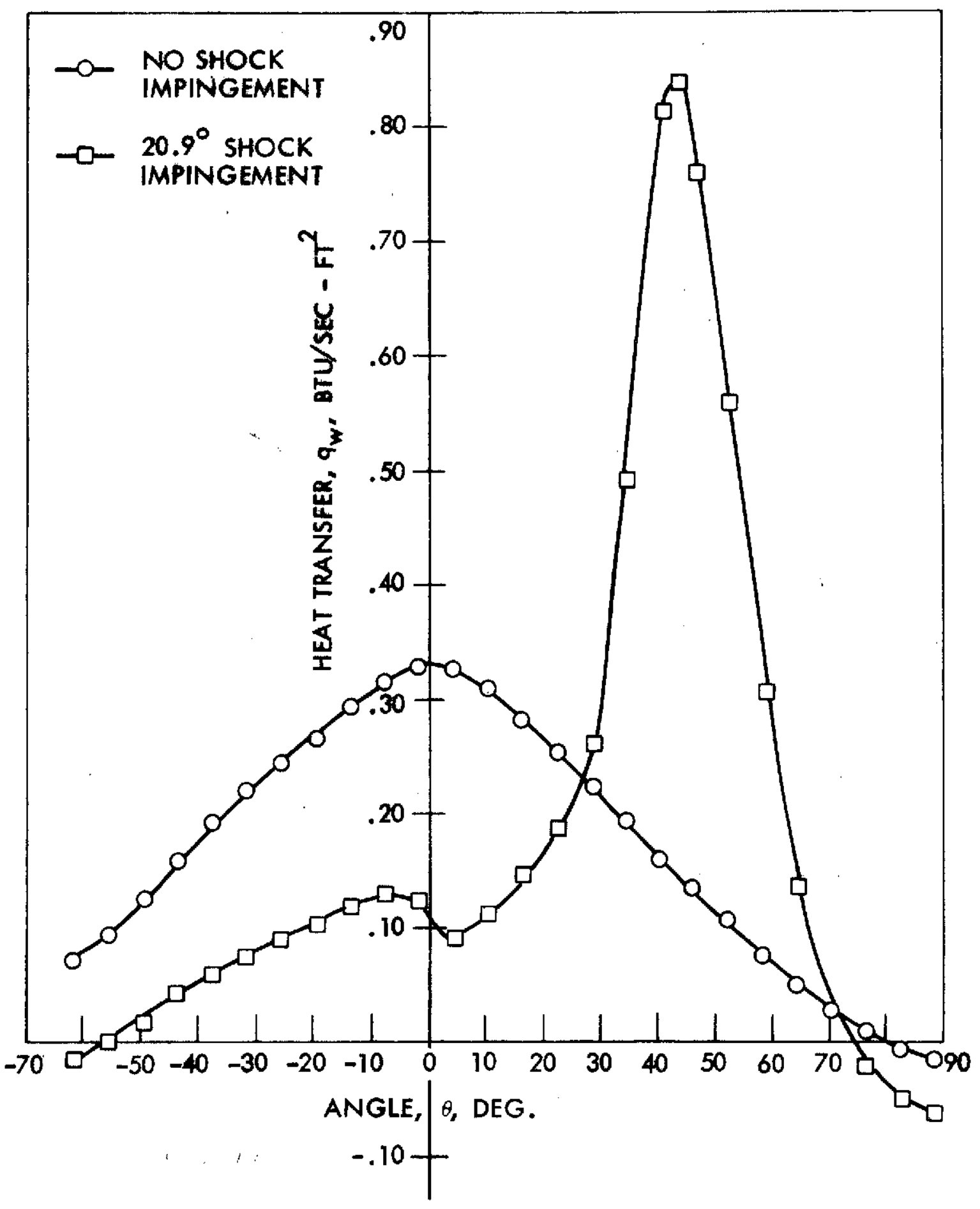

F1g. 6. Comparison of heat transfers. 